\title{
Radiating dipoles in photonic crystals
}

\author{
Kurt Busch ${ }^{1,2}$, Nipun Vats ${ }^{1}$, Sajeev John ${ }^{1}$ and Barry C. Sanders ${ }^{3}$ \\ 1 Department of Physics, University of Toronto, 60 St. George Street, Toronto, Ontario, Canada M5S 1A7 \\ 2 Institut für Theorie der Kondensierten Materie, Universität Karlsruhe, P.O. Box 6980, 76128 Karlsruhe, Germany \\ 3 Department of Physics, Macquarie University, Sydney, New South Wales, Australia 2109
}

(Revised: October 25, 2018)

\begin{abstract}
The radiation dynamics of a dipole antenna embedded in a Photonic Crystal are modeled by an initially excited harmonic oscillator coupled to a non-Markovian bath of harmonic oscillators representing the colored electromagnetic vacuum within the crystal. Realistic coupling constants based on the natural modes of the Photonic Crystal, i.e., Bloch waves and their associated dispersion relation, are derived. For simple model systems, well-known results such as decay times and emission spectra are reproduced. This approach enables direct incorporation of realistic band structure computations into studies of radiative emission from atoms and molecules within photonic crystals. We therefore provide a predictive and interpretative tool for experiments in both the microwave and optical regimes.
\end{abstract}

42.70.Qs,45.20.-d,45.20.Jj,45.30.+s

\section{INTRODUCTION}

Photonic crystals (PCs) have been the subject of intensive research over the past decade [1]. These are fabricated periodic dielectric arrays that employ a combination of (i) Mie scattering from individual elements of the array, and (ii) Bragg scattering from the periodic lattice, to induce a band structure for photon propagation. This band structure is, in many ways, analogous to electronic band structure in a semiconductor. Through a judicious selection of materials and of the periodicity of the lattice, the photonic dispersion relation and the associated electromagnetic (EM) mode structure of a PC can be adapted to a variety of device applications. The most dramatic modification of the photon dispersion occurs when the linear propagation of a photon in a PC is prohibited in all directions for a range of frequencies, giving rise to a complete photonic band gap (PBG).

The radiative dynamics of an optically active material placed within or near a PC can be dramatically modified from free space. This is a result of the "colored" electromagnetic reservoir provided by the solutions to the electromagnetic field equations within a PC. In the optical domain, theoretical studies of atomic transitions coupled to the EM modes of a PC with an optical PBG predict a number of novel quantum optical phenomena. These phenomena include: the suppression or enhancement of spontaneous emission and the associated fractional localization of light near radiating atoms [2.3]; rapid alloptical switching [4]; and anomalous superradiant emission, as well as low-threshold lasing near the edge of a PBG [5.6. . Microfabrication of PCs with complete PBGs at optical wavelengths has proven to be a difficult task, both because the lattice periodicity should be comparable to the wavelength of the light under consideration, and because a high dielectric contrast between the elements of the lattice is required. Fortunately, recent advances in microlithography [7] and in semiconductor in- filtration in colloidal crystals [8] have produced materials with significant pseudo-gaps in their photonic band structure 9]. The development of materials with complete PBGs in the optical regime appears imminent.

High-quality PBG materials at microwave frequencies have been available for some time 10. Sizeable bandgaps with center frequencies ranging from a few $\mathrm{GHz}$ up to $2 \mathrm{THz}$ have been reported; these crystals have thus proven the soundness of the concept of the PBG. Microwave PBG materials may be relatively easily manufactured using micro-machining techniques, and are currently of interest for applications such as the shielding of human tissue from microwave radiation, and for improving the radiation characteristics of microwave antennæ. Although PBG materials at microwave frequencies have been extensively studied, the behavior of radiating dipolar antennæ embedded in microwave PCs has not received the same degree of attention. This is despite the fact that such antennæ would share many properties in common with atomic emission in a PC. In the microwave domain, a dipole antenna could take the form of an electrically excited metallic pin with a high $Q$ (quality) factor.

The radiative dynamics of the above system can be described by a charged, one-dimensional simple harmonic oscillator (SHO). Such an electric dipole oscillator can also provide an excellent description of the radiation of single or multiple two-level atoms in the optical domain. This description is valid provided that the total excitation energy of the atoms is well below an energy where saturation (nonlinear) effects become important. Moreover, the radiation reservoir can itself be modeled as a bath of many independent SHOs: Radiative damping arises from a linear coupling between the system SHO and the large number of reservoir oscillator modes. The similarities between the microwave and optical systems, coupled with the mature state of microwave technology, suggests that many of the predicted effects for atomic 
dipoles in the optical domain could be realized and studied first in the microwave domain.

Analytical techniques exist for treating certain forms of coupling between the dipole and reservoir for certain modal distributions of the reservoir. However, PCs present coupling distributions and spectral properties which defy analytical methods. This is due to the presence of a restricted and rapidly-varying reservoir mode distribution, which renders invalid the usual BornMarkov type approximation schemes for the systemreservoir interaction. To obtain accurate results, we solve the system numerically for a large, but finite, number of oscillators in the reservoir by discretizing the modes of the reservoir following the approach of Ullersma [11]. In dealing with our system, there are crucial issues concerning obtaining the correct coupling strength between the oscillator and the reservoir modes, as well as in employing the proper renormalization and mode sampling in numerical simulations. When these criteria are satisfied, the SHO method comprises a powerful approach to treating radiative dynamics.

Here, we develop a rigorous quantitative treatment of the radiative dynamics of an electric dipole oscillator coupled to the electromagnetic reservoir within a model PC. In the process, we provide a sound theoretical basis for this and other approaches [12] to non-Markovian radiative dynamics which involve the discretization of a model electromagnetic reservoir. Additionally, we show how our method can be applied to realistic PC's with complicated dispersion relations and EM mode structures. The paper is organized as follows. In Section II, we develop a classical field theory for electromagnetic field modes in PCs, and we derive the coupling constants for the coupling between a radiating dipole and these Bloch modes. This leads to the Hamiltonian of the coupled system and the associated equations of motion. Renormalization issues arising from the non-relativistic nature of our theory are discussed in Section III, whereas Section IV describes the discretization of the reservoir and the numerical solution of the equations of motion. In Section V, these techniques are applied to a highly computationally challenging model, that of a three-dimensional, isotropic dispersion relation with a complete PBG. The demonstration of fractional localization and related phenomena validates the SHO approach to modeling radiative dynamics in PCs. In Section VI we summarize the results and emphasize the possibilities for testing these predictions experimentally in the microwave domain. The two appendices are concerned with the details of the field theory for the PC and with the details of the model of the one-sided, isotropic PBG, respectively.

\section{CLASSICAL FIELD THEORY}

In this section, we derive the equations governing the dynamics of a radiating dipole oscillator located inside a
PC. Typically the equation of motion for a damped oscillator, with time-dependent coordinate $q(t)$, is written as the second-order differential equation

$$
\ddot{q}(t)+\gamma \dot{q}(t)+\omega_{0}^{2} q(t)=F(t) .
$$

Here, we have introduced a damping constant $\gamma$, the natural frequency $\omega_{0}$ and the driving field $F(t)$ for the amplitude $q$ of the linear oscillator. For instance, for a freely oscillating $R L C$ circuit with ohmic resistance $R$, capacitance $C$ and inductance $L$, we have $\gamma=R / L$, $\omega_{0}^{2}=1 / L C, F(t)=0$, and $q(t)$ is the electric charge. Eq. (11) is, however, not the most general way of incorporating damping into the equations of motion for a harmonic oscillator. This description can break down if, for example, there is a suppression of modes in the reservoir to which the dipole oscillator can couple. Such a suppression of modes is a feature of the EM reservoir present in a PC. A more general description of damping forces acting on the harmonic oscillator therefore requires a precise knowledge of the mode structure of its environment, and the corresponding coupling of the system oscillator to these modes. In the case of a radiating dipole located in a PC, it is then appropriate to model its emission dynamics with a $\mathrm{SHO}$ coupled to a reservoir of SHOs. The essential difference between the vacuum and a PC is then contained in the spectral distribution, or density of states (DOS), of the reservoir oscillators, and in the coupling constants between the reservoir modes and the system oscillator.

The characterization of the reservoir is carried out in detail in Appendix A; here we only summarize the salient results. Given a radiating dipole with a natural frequency $\omega_{0}$, we obtain the classical Hamiltonian

$$
H=H_{\text {dip }}+H_{\text {res }}+H_{\text {ct }}+H_{\text {int }} .
$$

The first term on the right-hand side of the Hamiltonian is the energy of the dipole oscillator itself,

$$
H_{\mathrm{dip}}=\xi \omega_{0}|\alpha|^{2} \text {. }
$$

The natural frequency of the isolated oscillator is $\omega_{0}$, and $\xi$ is a constant with the dimension of energy $\times$ time. This permits us to write the energy of a SHO in units of its natural frequency $\omega$, i.e., $E(\omega)=\xi \omega$. The system oscillator's complex amplitude is given by the dimensionless, time-dependent quantity $\alpha$, defined with respect to the coordinate $q(t)$ of Eq. (1) as

$$
\alpha(t) \equiv \sqrt{\frac{L \omega_{0}}{2 \xi}} q(t)+\imath \sqrt{\frac{1}{2 \xi L \omega_{0}}}(L \dot{q}(t))
$$

The next term in the Hamiltonian (2) corresponds to the free evolution of the radiation reservoir, which is modeled as a bath of independent SHOs,

$$
H_{\mathrm{res}}=\sum_{\mu} \xi \omega_{\mu}\left|\beta_{\mu}\right|^{2}
$$


The natural electromagnetic modes of the $\mathrm{PC}$ are Bloch modes (see Appendix A), labeled with the index $\mu \equiv$ $(n \vec{k})$, where $n$ stands for the band index and $\vec{k}$ is a reciprocal lattice vector that lies in the first Brillouin zone (BZ). Their dispersion relation, $\omega_{\mu}$, is different from the vacuum case, and may have complete gaps and/or the corresponding density of states may exhibit appreciable pseudogap structure, the manifestation of multiple (Bragg) scattering effects in periodic media.

As we are working within the framework of a nonrelativistic field theory, we have introduced a mass renormalization counter term $H_{\mathrm{ct}}=-\xi \Delta|\alpha|^{2}$ that cancels unphysical UV-divergent terms [13,14]. The quantity $\Delta$ is specified in Section III.

The interaction between the oscillator and the reservoir is given by a linear coupling term. As the oscillator frequency is quite large, and the effective linewidth of the oscillation is relatively small, it is possible to simplify the interaction by applying the rotating-wave approximation. In this approximation, couplings in the Hamiltonian of the form $\alpha^{*} \beta_{\mu}^{*}$ and its complex conjugate are neglected, as these terms oscillate very rapidly compared to the terms of the type $\alpha^{*} \beta_{\mu}$ and its conjugate. Hence, the interaction Hamiltonian can be expressed as

$$
H_{\mathrm{int}}=-\imath \xi \sum_{\mu}\left(\alpha^{*} g_{\mu}^{*} \beta_{\mu}-\alpha g_{\mu} \beta_{\mu}^{*}\right) .
$$

In the case of a point dipole, i.e., when its spatial extent $a$ is much smaller than the wavelength corresponding to its natural frequency, $\lambda_{0}=2 \pi \omega_{0} / c$, the coupling constants $g_{\mu}$ can be derived from (i) the magnitude of the dipole moment, $d(t)=a q(t)$, located at $\overrightarrow{r_{0}}$, and (ii) the dipole orientation, $\hat{d}$, relative to that of the Bloch modes, $\vec{E}_{\mu}\left(\vec{r}_{0}\right)$ :

$$
g_{\mu} \equiv g_{\mu}\left(\vec{r}_{0}\right)=a c \sqrt{\frac{\pi}{L \omega_{0} \omega_{\mu}}}\left(\hat{d} \cdot \vec{E}_{\mu}^{*}\left(\vec{r}_{0}\right)\right) .
$$

This dependence of the coupling constant on the dipole's precise location within the $\mathrm{PC}$ is the second essential difference from the free-space case. As shown in Refs. [15,16, this position dependence may be quite strong, thus making its incorporation a sine qua non for any quantitative theory of of radiating antennæ or fluorescence phenomena in realistic PCs.

The emission dynamics can be evaluated from the Poisson brackets of the oscillator amplitudes and their initial values, $\alpha(0)=1$ and $\beta_{\mu}(0)=0(\forall \mu)$. Our choice of $\alpha(0)$ and $\beta_{\mu}(0)$ corresponds to the initial condition of an excited dipole antenna and a completely de-excited bath. The only non-zero Poisson brackets are

$$
\left\{\alpha, \alpha^{*}\right\}=\left\{\beta_{\mu}, \beta_{\mu}^{*}\right\}=\frac{\imath}{\xi} .
$$

Eqs. (2), (7) and (8), together with the initial values for the oscillator amplitudes, completely determine the emission dynamics of a radiating dipole embedded in a
PC. In the following sections, we solve the corresponding equations of motion. This task is complicated by the nature of the reservoir's excitation spectrum: as discussed, the non-smooth density of states prohibits the use of a Markovian approximation and its appealing simplifying features [2,3,6]. Instead, we have to revert to a solution of the full non-Markovian problem. This is accomplished by firstly rearranging the reservoir modes in a manner more suitable to both analytical as well as numerical solutions, and subsequently solving the equations of motion. In what follows, we bridge the gap between previous studies of simplified model dispersion relations [2,3,6] and band structure computations [15,17].

Although we will formally develop our theory for an LC circuit in a microwave PC, we emphasize that the formalism applies equally well to a semiclassical Lorentz oscillator model of an excited two-level atom, i.e., an electron with charge $e$ and mass $m$ which is bound to a stationary nucleus, for which the energy of excitation is well below that required for saturation effects to become relevant. The oscillator coordinate $q(t)$ may then be identified with the deviation of the electron's position from its equilibrium value, $\gamma$ is the inverse life time of the excited state, and $\omega_{0}$ denotes the frequency for transitions between excited and ground state of the two-level atom. This corresponds to making the substitutions:

$$
L \rightarrow m, \quad(L \dot{q}) \rightarrow p, \quad \xi \rightarrow \hbar,
$$

where $h=2 \pi \hbar$ is Planck's constant.

\section{PROJECTED LOCAL DENSITY OF STATES, MASS RENORMALIZATION AND LAMB SHIFT}

From the Hamiltonian (2) we derive the equations of motion for the amplitudes

$$
\begin{aligned}
\dot{\alpha}(t) & =-\imath\left(\omega_{0}-\Delta\right) \alpha(t)-\imath \xi \sum_{\mu} g_{\mu}^{*} \beta_{\mu}(t) \\
\dot{\beta}_{\mu}(t) & =-\imath \omega_{\mu} \beta_{\mu}(t)+g_{\mu} \alpha(t),
\end{aligned}
$$

for which we seek a solution with initial conditions $\alpha(0)=$ 1 and $\beta_{\mu}(0)=0(\forall \mu)$. Our formalism however requires that we first determine the mass renormalization counter term $\Delta$. This is most conveniently done in a rotating frame with slowly varying amplitudes $a(t)$ and $b(t)$, defined as $\alpha(t)=a(t) e^{-\imath \omega_{0} t}$ and $\beta(t)=b(t) e^{-\imath \omega_{\mu} t}$ respectively:

$$
\begin{aligned}
\dot{a}(t) & =-\imath \xi \sum_{\mu} g_{\mu}^{*} e^{\imath\left(\omega_{0}-\omega_{\mu}\right) t} b_{\mu}(t)+\imath \Delta a(t) \\
\dot{b}(t) & =g_{\mu} e^{-\imath\left(\omega_{o}-\omega_{\mu}\right) t} a(t) .
\end{aligned}
$$

Conversely, Eqs. (12) and (13) comprise a stiff set of differential equations making their solution a difficult task. Numerical solution of the problem is more easily performed in the non-rotating frame, to which we return in Sect. IV. 
Eq. (13) may be formally integrated,

$$
b_{\mu}(t)=g_{\mu} \int_{0}^{t} d t^{\prime} e^{-\imath\left(\omega_{0}-\omega_{\mu}\right) t^{\prime}} a\left(t^{\prime}\right),
$$

and inserted into Eq. (12) to yield

$$
\dot{a}(t)=-\int_{0}^{\infty} d t^{\prime} G\left(t-t^{\prime}\right) a\left(t^{\prime}\right)+\imath \Delta a(t),
$$

where the Green function $G(\tau)$ contains all the information about the reservoir and is the subject of our studies for the remainder of this section. It is defined as

$$
G(\tau) \equiv \Theta(\tau) \sum_{\mu}\left|g_{\mu}\right|^{2} e^{\imath\left(\omega_{0}-\omega_{\mu}\right) \tau} .
$$

Here, $\Theta(\tau)$ denotes the Heaviside step function, which ensures the causality of $G(\tau)$. We now proceed to evaluate $G(\tau)$ for the form of the coupling constants $g_{\mu}$ given in Eq. (7). To this end, we introduce the projected local DOS (PLDOS) $N\left(\vec{r}_{0}, \hat{d}, \omega\right)$ through

$$
N\left(\vec{r}_{0}, \hat{d}, \omega\right)=\sum_{n} \int_{\mathrm{BZ}} \frac{d^{3} k}{(2 \pi)^{3}} \delta\left(\omega-\omega_{n \vec{k}}\right)\left|\hat{d} \cdot \vec{E}_{n \vec{k}}\left(\vec{r}_{0}\right)\right|^{2},
$$

where we have replaced the symbolic sum over $\mu$ by its proper representation as a sum over bands plus a wave vector integral over the BZ. With these changes, we may rewrite $G(\tau)$ compactly as

$$
G(\tau)=\beta \Theta(\tau) \int_{0}^{\infty} d \omega \frac{N\left(\vec{r}_{0}, \hat{d}, \omega\right)}{\omega} e^{\imath\left(\omega_{0}-\omega\right) \tau} .
$$

Here, we have abbreviated $\beta=\left(\pi a^{2} c^{2}\right) /\left(L \omega_{0}\right)$. Eq. (18) makes more explicit what we have argued before: The spontaneous emission dynamics of active media in Photonic Crystals are completely determined by the PLDOS, $N\left(\vec{r}_{0}, \hat{d}, \omega\right)$. As the PLDOS may be drastically different from location to location within the Wigner-Seitz cell of the PC [15,16], it is imperative to have detailed knowledge about where in the $\mathrm{PC}$ the dipole is situated in order to understand and predict the outcome of corresponding experiments.

One additional point deserves special attention: the total DOS, $N(\omega)$, is related to the local DOS via

$$
\begin{aligned}
N(\omega) & =\frac{1}{V} \int_{V} d^{3} r \int d \Omega_{\hat{d}} \epsilon_{p}(\vec{r}) N(\vec{r}, \hat{d}, \omega) \\
& \neq \frac{1}{V} \int_{V} d^{3} r \int d \Omega_{\hat{d}} N(\vec{r}, \hat{d}, \omega),
\end{aligned}
$$

where $V$ is the volume of the Wigner-Seitz cell, and $\int d \Omega_{\hat{d}}$ is the average over all possible orientations of the dipole. Strictly speaking, it is not possible to base conclusions about the radiation dynamics on the total DOS. This is a direct consequence of the fact that the natural modes of PCs are Bloch waves rather than plane waves as in free space. Depending on the band index, these Bloch modes prefer to "reside" predominantly in either low or high dielectric index regions (so-called air and dielectric bands respectively). Only in the case of very low index contrast ("nearly free photons") may the total DOS be viewed as a reliable guide to interpreting radiative dynamics within a PC. The total DOS is, nevertheless, an adequate rule-of-thumb estimator.

From Eq. (17) we can now obtain the Fourier transform of the Green function, $G\left(\Omega-\omega_{0}\right)$, centered around the atom's bare transition frequency $\omega_{0}$ :

$$
\begin{aligned}
G\left(\Omega-\omega_{0}\right)= & \int_{0}^{\infty} d t e^{\imath\left(\Omega-\omega_{0}\right) t} G(t) \\
= & \pi \beta \frac{N\left(\vec{r}_{0}, \hat{d}, \Omega\right)}{\Omega} \Theta(\Omega) \\
& +\imath \beta \int_{0}^{\infty} d \omega \frac{N\left(\vec{r}_{0}, \hat{d}, \omega\right)}{\omega} \wp\left(\frac{1}{\Omega-\omega}\right),
\end{aligned}
$$

where $\wp$ stands for the principal value.

For large $\omega$, we have $N\left(\vec{r}_{0}, \hat{d}, \omega\right) \propto \omega^{2}$. The imaginary part of $G\left(\Omega-\omega_{0}\right)$ apparently contains a linear divergence in the UV. This divergence is to be expected for a nonrelativistic theory, analogous to the problem of spontaneous emission in vacuum [13], and is removed from the theory by using the mass counter renormalization term $\Delta$, as first pointed out by Bethe [14. Consequently, we decompose the imaginary part of $G\left(\Omega-\omega_{0}\right)$ into

$$
\Im\left(G\left(\Omega-\omega_{0}\right)\right) \simeq-\left(\Delta+\delta_{\mathrm{vac}}+\delta_{a}\right),
$$

where we have used the notation:

$$
\begin{aligned}
& \Delta=\beta \int_{0}^{\infty} d \omega \frac{N\left(\vec{r}_{0}, \hat{d}, \omega\right)}{\omega^{2}} \\
& \delta_{\mathrm{vac}}=-\frac{\beta \omega_{0}}{\pi^{2} c^{3}} \int_{0}^{\Omega_{c}} d \omega \wp\left(\frac{1}{\omega_{0}-\omega}\right) \\
& \delta_{\mathrm{a}}=-\frac{\beta \omega_{0}}{\pi^{2} c^{3}} \int_{0}^{\Omega_{c}} d \omega \wp\left(\frac{1}{\omega_{0}-\omega}\right) \times \\
& \quad \times \frac{N\left(\vec{r}_{0}, \hat{d}, \omega\right)-N^{(\mathrm{vac})}(\omega)}{\omega^{2}} .
\end{aligned}
$$

Here, we have performed a Wigner-Weisskopf-type approximation on the vacuum and anomalous Lamb shifts [2], $\delta_{\mathrm{vac}}$ and $\delta_{\mathrm{a}}$, respectively. This approximation is justified by the fact that, despite its highly non-Markovian nature, a radiating dipole in a PC is still a weak coupling problem, as can be seen, for instance, by estimating the coupling constant

$$
g \simeq d_{0} \omega_{0} \sqrt{\frac{2 \pi}{V \xi \omega_{n \vec{k}}}}
$$

in the Lorentz oscillator model. Here, $V \approx \bar{a}^{3}$ is the volume of the Wigner-Seitz cell of the PC ( $\bar{a}$ is the corresponding lattice constant) and $d_{0}=e a_{0}$ is the oscillator's dipole moment for the elementary charge $e$ and 
Bohr atomic radius, $a_{0}$. At optical frequencies $\left(\omega \approx 10^{15}\right.$ $\left.\mathrm{s}^{-1}\right)$, a silicon inverted opal has a PBG at the frequency $\bar{a} \omega / 2 \pi c \approx 0.8$, so that we obtain $10^{-7} \leq g / \omega_{0} \leq$ $10^{-6} \ll 1$, thus justifying our Wigner-Weisskopf approximation. As a consequence, we must treat the real part of $G\left(\Omega-\omega_{0}\right)$ exactly, but are still allowed to tackle the imaginary part of $G\left(\Omega-\omega_{0}\right)$ using standard perturbation methods of QED. In addition, we have introduced the vacuum or free-space DOS $N^{(\text {vac })}(\omega)=\omega^{2} /\left(\pi^{2} c^{3}\right)$, and a cutoff frequency $\Omega_{c} \gg \omega_{0}$, which is chosen large enough that the results of the following analysis remain independent of the precise value of $\Omega_{c}$. In a Lorentz oscillator model, for instance, $\Omega_{c}$ can be identified with the Compton frequency $\Omega_{c} \simeq m c^{2} / \hbar$, as $\omega>\omega_{c}$ probes the relativistic aspects of the oscillating charge, which are beyond the scope of the model.

With the foregoing analysis, we have determined the mass renormalization counter term $\Delta$. In addition, we have derived an explicit expression for the anomalous Lamb shift $\delta_{a}$ [2] which originates in the "reshuffling" of the reservoir's spectral weight by the PC.

\section{DISCRETIZATION OF THE RESERVOIR}

To solve the equation of motion for the amplitude of the system oscillator, let us rewrite Eq. (15) in a more explicit form:

$$
\begin{aligned}
\dot{a}(t)= & -\int_{0}^{\infty} d \omega N\left(\vec{r}_{0}, \hat{d}, \omega\right) g^{2}(\omega) \int_{0}^{t} d t^{\prime} e^{\imath\left(\omega_{0}-\omega\right)\left(t-t^{\prime}\right)} a\left(t^{\prime}\right) \\
& +\imath \Delta a(t),
\end{aligned}
$$

where $g^{2}(\omega)=\beta / \omega$, and the mass renormalization counter term $\Delta$ is given by

$$
\Delta=\beta \int_{0}^{\infty} d \omega \frac{N\left(\vec{r}_{0}, \hat{d}, \omega\right)}{\omega^{2}}
$$

We remind the reader that $a(0)=1$.

We are now in a position to comment on the origin of the linear damping term $\gamma \dot{q}(t)$ that appears in Eq. (11): If we consider the long time limit, i. e., $t \gg 1 / \omega_{0}$, and assume that the PLDOS $N\left(\vec{r}_{0}, \hat{d}, \omega\right)$ is a smooth function for frequencies around $\omega_{0}$, we can approximate the frequency integral in Eq. (21) by $\left[2 \pi \beta N\left(\vec{r}_{0}, \hat{d}, \omega_{0}\right) / \omega_{0}\right] \delta(t-$ $\left.t^{\prime}\right)$, which leads to

$$
\dot{a}(t)=-\gamma a(t)
$$

where the decay constant is defined as

$$
\gamma=2 \pi \beta N\left(\vec{r}_{0}, \hat{d}, \omega_{0}\right) / \omega_{0}
$$

This approximation is is valid only for long times relative to $1 / \omega_{0}$, and for a sufficiently smooth density of states. However, in the case of a PC, the PLDOS may have sharp discontinuities and gaps, thus requiring that the full equations of motion instead.

To solve the integro-differential equation (21) in a PC, we appeal to the literal meaning of the PLDOS as a density of states: $N\left(\vec{r}_{0}, \hat{d}, \omega\right)$ may be interpreted as an unnormalized probability density of finding a reservoir oscillator with frequency $\omega$ at position $\vec{r}_{0}$ and orientation $\hat{d}$. Consequently, we transform Eq. (21) back to a system of coupled differential equations by employing a Monte Carlo integration scheme for an arbitrary function $f(\omega)$ according to

$$
\begin{aligned}
\int_{0}^{\infty} d \omega N\left(\vec{r}_{0}, \hat{d}, \omega\right) f(\omega) & \simeq \int_{0}^{\Omega_{c}} d \omega N\left(\vec{r}_{0}, \hat{d}, \omega\right) f(\omega) \\
& \approx \frac{N_{0}}{M} \sum_{i=1}^{M} f\left(\omega_{i}\right)
\end{aligned}
$$

where the normalization constant

$$
N_{0}=\int_{0}^{\Omega_{c}} d \omega N\left(\vec{r}_{0}, \hat{d}, \omega\right)
$$

depends on the cutoff frequency, $\Omega_{c}$. There are $M \gg 1$ bath oscillators, contained within a set of frequencies $\left\{\omega_{i}, 1 \leq i \leq M\right\}$, the frequencies of which are obtained by randomly sampling the interval $\left[0, \Omega_{c}\right]$ according to the probability density $p\left(\vec{r}_{0}, \hat{d}, \omega\right)=N\left(\vec{r}_{0}, \hat{d}, \omega\right) / N_{0}$. Note that the $\omega_{i}$ may be degenerate, as prescribed by $p\left(\vec{r}_{0}, \hat{d}, \omega\right)$.

Applying this Monte Carlo scheme to Eq. (21) and transforming back to a non-rotating frame in order to avoid having to solve a numerically stiff problem, we obtain

$$
\begin{aligned}
& \dot{\alpha}(t)=-\imath\left(\omega_{0}-\Delta\right) \alpha(t)-\imath \xi \sum_{i=1}^{N} g_{i} \beta_{i}(t) \\
& \dot{\beta}_{i}(t)=-\imath \omega_{i} \beta_{i}(t)+g_{i} \alpha(t),
\end{aligned}
$$

where $g_{i}=g\left(\omega_{i}\right), 1 \leq i \leq M$, and the mass renormalization counter term is evaluated up to the cutoff frequency $\Omega_{c}$, i.e., $\Delta=\int_{0}^{\Omega_{c}} d \omega N\left(\vec{r}_{0}, \hat{d}, \omega\right) / \omega^{2}$.

When comparing Eqs. (27) and (28) to our initial equations of motion, Eqs. (10) and (11), we observe that the considerations in the previous section have allowed us to rearrange the three-dimensional wave vector sum over the modes $\mu \equiv(n \vec{k})$ into a simple one-dimensional sum over a set of frequencies $\left\{\omega_{i}\right\}$ with a probability distribution $p\left(\vec{r}_{0}, \hat{d}, \omega\right)$ that is easily determined through standard photonic band structure computation [15]. In the following section, we give the solutions of (27) and (28) for a model system which has previously been treated by other methods. In particular, we will demonstrate that known results for the radiative dynamics can be recaptured and do not depend on the the value of the cutoff frequency $\Omega_{c}$ and the number $M$ of reservoir oscillators once these quantities are large enough such that all the relevant features of $N\left(\vec{r}_{0}, \hat{d}, \omega\right)$, are adequately represented. 


\section{NUMERICAL RESULTS FOR A MODEL SYSTEM}

In order to establish the validity of our approach, we now solve Eqs. (27) and (28) for a generic model of a PBG, the three-dimensional isotropic, one-sided PBG [3]. In Appendix B, we outline the construction of the model's dispersion relation and how to obtain the corresponding model DOS, $N_{m}(\omega)$. We note that we do not appeal to an effective mass approximation in the dispersion relation [6], as is done in most treatments of band-edge dynamics. This allows us to recover the correct form of the large frequency behavior of the photon density of states.

In Fig. 1, we show the behavior of $N_{m}(\omega)$ as a function of frequency for values of the relevant parameters, the gap size parameter $\eta=0.8$ and the normalized center frequency $\omega_{c} a / 2 \pi c=0.5$ (see Appendix B). The DOS exhibits a square-root singularity at the band edge $\omega_{u} a / 2 \pi c=0.6$, as well as a UV divergence, $N_{m}(\omega) \propto \omega^{2}$, as $\omega \rightarrow \infty$; these are the characteristic features of this model. Due to the simultaneous presence of both divergences, this model clearly represents a severe numerical test of our approach. In order to test the method, we thus replace the PLDOS entering Eqs. (27) and (28) by $N_{m}(\omega)$.

In Fig. 2, we present the results of our numerical solution for the radiation dynamics of a dipole oscillator with frequency $\omega_{0}$ that is coupled to the modes of a PC, as described by Eqs. (27) and (28), for various values of the bare oscillator frequency, $\omega_{0} a / 2 \pi c$, relative to the bandedge at $\omega_{u} a / 2 \pi c=0.6$. The coupling strength has been chosen such that $g\left(\omega_{0}\right)=10^{-4}$, corresponding to $\beta=10^{-8} \times \omega_{0}^{3}$.

Clearly visible are normal mode oscillations, also referred to as vacuum Rabi oscillations, and the fractional localization of the oscillator's energy at long times near the photonic band-edge [3]. As expected, for frequencies deep in the photonic band-gap $\left(\omega_{0} a / 2 \pi c=0.58\right)$, where the system oscillator is effectively decoupled from the bath oscillators, we find no noticeable decay of the oscillator amplitude. Deep in the photonic conduction band $\left(\omega_{0} a / 2 \pi c=0.62\right)$, the system oscillator is coupled to a bath with a smooth and slowly-varying mode density, as in free space. We therefore observe exponential decay of the oscillator amplitude, though with a time scale that differs significantly from that in free space. Due to the large value of the DOS close to the photonic band edge, the initial decay is faster for bare oscillator frequencies close to this edge than for frequencies deep inside the allowed photonic band. These results were obtained for a smooth exponential cutoff for the DOS around $\Omega_{c} a / 2 \pi c=3.0$ and $M=2.5 \times 10^{5}$ oscillators representing the modes of the PC. We also performed numerical simulations between all combinations of $\Omega_{c}$ and $M$ with values $\Omega_{c} a / 2 \pi c=3.0,6.0,9.0$ and $M=2.5 \times 10^{5}, 5 \times 10^{5}, 10^{6}$ and found that the numerical values differ by at most $0.2 \%$ of the values shown in
Fig 1. This demonstrates that, despite the presence of the singularities in the DOS, our approach still provides accurate and convergent results.

\section{DISCUSSION}

In summary, we have developed a realistic field theory for an oscillating electric dipole located in a PC. The theory is based on the natural modes of the PC, the Bloch waves, and allows the direct incorporation of realistic band structure calculations in order to obtain quantitative results for the radiation dynamics of the dipole antenna. We have shown how the theory must be renormalized in order to account for unphysical divergences and have identified the classical analogue of the Lamb shift of the dipole's natural radiation frequency. Finally, we have developed a reliable numerical scheme based on a probability interpretation of the PLDOS that solves the equations of motion for the dipole oscillator coupled to the electromagnetic mode reservoir of the PC.

The viability of this approach was demonstrated for an isotropic model DOS for which we have derived wellknown results for radiating atomic systems 33 in the context of a radiating classical dipole. The model considered contains two divergences, one square-root-divergence at the photonic band edge and a quadratic UV-divergence, and therefore clearly comprises the most serious test of our approach. More realistic models of a three dimensional photonic band-edge take into account the anisotropy of the BZ, and therefore do not suffer from a band-edge singularity [6]. As a result, our formalism is clearly more than capable of treating more realistic descriptions of the electromagnetic reservoir within a PC.

Though we have developed our theory for an LC circuit in a microwave PC, we have pointed out in Section II that the formalism applies equally well to a semiclassical Lorentz oscillator model of an excited two-level atom. Therefore, our approach is applicable to both microwave antennæ and to optical atomic transitions. However, technological constraints suggest that microwave experiments will likely be easier to perform than optical experiments involving single atoms. As discussed, an appropriate microwave antenna could, for example, take the form of a high-Q metallic pin placed in or near a PC. The pin can then be excited by a focused ultrashort laser pulse that generates free carriers at one end; these carriers then undergo several oscillations across the pin before re-establishing charge equilibrium. The resulting signal could be easily detected and compared with the emission from such an antenna positioned in free space, or within a homogeneous sample of the dielectric material that makes up the backbone of the PC under consideration.

In its own right, such a microwave system could have considerable applications in radio science and microwave technology. For example, the PBG can be used as a fre- 
quency filter, and can be used to fine tune the bandwidth of a dipole emitter with a resonant frequency near the edge of the gap. It may also be possible to actively modify the photonic band structure, effectively changing the radiation pattern of a dipole emitter. A feasible scheme for active band structure modification has recently been proposed in the context of optical PCs [18], in which the PC is infiltrated with a liquid crystalline material whose nematic director is aligned using applied electric fields. By rotating the director, it was found that the band structure could be significantly modified, and that PBGs may be opened and closed altogether. Similar methods may be applied to the case of microwave PCs.

Although we have concentrated specifically on the linear model, the method of coupled oscillators can be extended to treat a nonlinear antennæ, or a collection of two-level atoms in a regime where saturation effects arise. As we have shown here, this method of coupled classical oscillators reproduces effects normally associated with quantum optical calculations. We expect that a nonlinear oscillator model will reproduce some of the effects studied for a single two-level atom coupled to the modes of a PC without the need for quantizing the field. However, a classical treatment would need to be abandoned if multiphoton excitations are non-negligible [12. Given that multiphoton effects are difficult to observe in the microwave domain $\sqrt{19}$ and even more challenging in the optical domain [20], it is reasonable to expect that a classical model of radiative dynamics in a PC should be sufficient for foreseeable experiments.

\section{ACKNOWLEDGMENTS}

We are grateful to K.-J. Boller and R. Beigang for stimulating discussions concerning the experimental realization of radiating dipoles in microwave PCs. The work of KB the was supported by the Deutsche Forschungsgemeinschaft (DFG) under Grant Bu 1107/1-1. NV acknowledges support from the Ontario Graduate Scholarship Program. BCS acknowledges support from the Department of Physics, University of Toronto, and the support of an Australian Research Council Large Grant. This work was supported in part by the New Energy and Industrial Technology Development Organization of Japan and by Photonics Research Ontario.

\section{APPENDIX A: CLASSICAL FIELD THEORY FOR PHOTONIC CRYSTALS}

In this Appendix, we present a self-contained formulation of a classical field theory for the Bloch modes of a PC, and we develop the Hamiltonian describing the coupling of a radiating dipole couples to these modes. As a first step, we review the computation of dispersion relations, and of electric and magnetic field modes from band structure calculations 157. We then demonstrate how the results of such band structure calculations can be used to construct the corresponding vector potentials and free field Hamiltonian. Finally, we derive the full minimal coupling Hamiltonian for a classical radiating dipole embedded in a PC. This may be compared to the formulation of a general, quantized field theory for EM modes in nonuniform dielectric media in terms of a normal mode expansion in the context of quantum optics [21.

\section{Review of band structure calculations}

We develop our theory in terms of the magnetic field $\vec{H}$ rather than in terms of the electric or displacement fields because (i) $\nabla \cdot \vec{H}=0$ and, (ii) the transverse and longitudinal components of the magnetic field are continuous across the dielectric boundaries. This leads to more rapid convergence of the relevant Fourier series expansions.

In a three-dimensional PC, we can write the eigenvalue equation for the magnetic field $\vec{H}$ as

$$
\nabla \times\left(\eta_{p}(\vec{r}) \nabla \times \vec{H}\right)+\frac{\omega^{2}}{c^{2}} \vec{H}=\overrightarrow{0}
$$

with $\eta_{p}(\vec{r})$ the inverse of the periodic dielectric permittivity,

$$
\epsilon_{p}(\vec{r})=\epsilon_{b}+\left(\epsilon_{a}-\epsilon_{b}\right) \sum_{\vec{n} \in \mathcal{Z}^{3}} S(\vec{r}-\vec{n} \cdot \boldsymbol{A}) .
$$

The medium is assumed to consist of a background material with bulk permittivity $\epsilon_{b}$ and a set of scatterers, with bulk permittivity $\epsilon_{a}$. The shape of the scatterers is described by the function $S$, i. e.,, $S(\vec{r})=1$ if $\vec{r}$ lies inside the scatterer and zero elsewhere, distributed periodically at positions

$$
\{\vec{R}\}=\left\{\sum_{i=1}^{3} n_{i} \vec{a}_{i} \mid n_{i} \in \mathcal{Z}\right\} .
$$

The notation of Eq. A2 is obtained by defining the matrix $\boldsymbol{A}=\left(\vec{a}_{1} \vec{a}_{2} \vec{a}_{3}\right)$ and $\mathcal{Z}^{3}=\mathcal{Z} \otimes \mathcal{Z} \otimes \mathcal{Z}$. The dielectric permittivity is spatially periodic modula $\vec{n} \cdot \boldsymbol{A}$. The assumption of a scalar permittivity is reasonable for bulk materials which are not birefringent but in no way restricts the considerations below. Chromatic dispersion effects are considered to be negligible, thus allowing the time-dependence of the permittivity to be ignored. Let us define the dual matrix $\boldsymbol{B}=2 \pi\left(\boldsymbol{A}^{-1}\right)^{T}$. For $\boldsymbol{B}=\left(\vec{b}_{1} \vec{b}_{2} \vec{b}_{3}\right)$, this definition leads to the orthogonality relation

$$
\vec{a}_{i} \cdot \vec{b}_{j}=2 \pi \delta_{i j}
$$

Whereas the points $\vec{n} \cdot \boldsymbol{A}$ are the real space lattice vectors, the points $\vec{m} \cdot \boldsymbol{B}$, for $\vec{m} \in \mathcal{Z}^{3}$ are the reciprocal lattice 
vectors. The inverse permittivity can be expanded in the dual basis as

$$
\eta_{p}(\vec{r})=\sum_{\vec{m} \in \mathcal{Z}^{3}} \eta_{\vec{m}} e^{\imath \vec{m} \cdot \boldsymbol{B} \cdot \vec{r}} .
$$

The differential equation A1 has periodic coefficients. By the Bloch-Floquet theorem we can expand the magnetic field as

$$
\vec{H}_{\vec{k}}=e^{\imath \vec{k} \cdot \vec{r}} \vec{u}_{\vec{k}}(\vec{r})
$$

where $\vec{u}_{\vec{k}}$ is spatially periodic modulo $\boldsymbol{A}$; that is,

$$
\vec{u}_{\vec{k}}(\vec{r})=\vec{u}_{\vec{k}}(\vec{r}+\vec{n} \cdot \boldsymbol{A})
$$

The set $\{\vec{k}\}$ labeling the solutions can be restricted to lie within in the irreducible part of the first Brillouin zone (BZ), since any value of $\vec{k}$ can then be obtained through a combination of group transformations with respect to an operation from the point group of the crystal and translations with respect to a reciprocal lattice vector. We can therefore express each wavevector $\vec{k}$ as

$$
\vec{k} \equiv \vec{k}_{\boldsymbol{T}, \vec{m}} \equiv \vec{k}_{*} \cdot \boldsymbol{T}+\vec{m} \cdot \boldsymbol{B},
$$

where $\vec{k}_{*}$ is an element of the irreducible part of the 1 . BZ and $\boldsymbol{T}$ an element of the crystal's point group.

Applying the Bloch-Floquet theorem, Eq. (A6), the magnetic field can be expanded as

$$
\vec{H}_{\vec{k}}=e^{\imath \vec{k} \cdot \vec{r}} \sum_{\vec{m}} \sum_{\lambda=1}^{2} h_{\vec{m}}^{\vec{k}, \lambda} \hat{e}_{\vec{m}}^{\vec{k}, \lambda} e^{\imath \vec{m} \cdot \boldsymbol{B} \cdot \vec{r}} .
$$

Here $\lambda$ is the index of polarization and the vectors

$$
\left\{\hat{e}_{\vec{m}}^{\vec{k}, 1}, \hat{e}_{\vec{m}}^{\vec{k}, 2}, \frac{\vec{k}+\vec{m} \cdot \boldsymbol{B}}{|\vec{k}+\vec{m} \cdot \boldsymbol{B}|}\right\}
$$

form an orthonormal right-handed triad. This expansion inserted into Eq (A1) yields an infinite eigenvalue problem which is then solved numerically by a suitable truncation. Typically the cardinality of the set $\{\vec{m}\}$ is on the order of $10^{3}$ 15. For any given $\vec{k}_{*}$ we obtain a discrete set of eigenfrequencies $\omega_{n \vec{k}}$ and corresponding eigenfunctions $H_{n \vec{k}}$ which we label by the band index $n \in \mathcal{N}$. It is important to note that the expression for the electric field can be recovered from the magnetic field via

$$
\vec{E}_{n \vec{k}}(\vec{r})=-i \frac{c}{\omega_{n \vec{k}} \epsilon_{p}(\vec{r})} \nabla \times \vec{H}_{n \vec{k}}(\vec{r})
$$

In addition, the Bloch waves obey the following orthogonality relations:

$$
\begin{array}{r}
\int d^{3} r \vec{H}_{n \vec{k}}^{*}(\vec{r}) \cdot \vec{H}_{m \vec{k}^{\prime}}(\vec{r}) \propto \delta_{n m} \delta\left(\vec{k}-\vec{k}^{\prime}\right), \\
\int d^{3} r \epsilon_{p}(\vec{r}) \vec{E}_{n \vec{k}}^{*}(\vec{r}) \cdot \vec{E}_{m \vec{k}^{\prime}}(\vec{r}) \propto \delta_{n m} \delta\left(\vec{k}-\vec{k}^{\prime}\right),
\end{array}
$$

where the integration is over all space in both cases. We are free to choose the constants of proportionality in the above relations, and do so in the next subsection.

\section{Free-field Hamiltonian}

Based on the above considerations, we are now in a position to derive the general expressions for the scalar and vector potential, $\phi(\vec{r}, t)$ and $\vec{A}(\vec{r}, t)$ respectively, for the classical Hamiltonian of the free radiation field. We find that the expressions become particularly transparent in the Dzyaloshinsky gauge, i.e., when $\phi(\vec{r}, t) \equiv 0$. Then,

$$
\begin{aligned}
& \vec{E}(\vec{r}, t)=-\frac{1}{c} \frac{\partial \vec{A}(\vec{r}, t)}{\partial t}, \\
& \vec{H}(\vec{r}, t)=\nabla \times \vec{A}(\vec{r}, t),
\end{aligned}
$$

and the gauge condition $\nabla \cdot\left(\epsilon_{p}(\vec{r}) \vec{A}(\vec{r}, t)\right)=0$, reveals that in a PC the natural modes of the radiation field are no longer transverse. This is of importance when quantizing the field theory 21,22]. Given Eqs. (A1), (A11), (A14) and (A15), it is now straightforward to derive the following expansion of the vector potential $\vec{A}(\vec{r}, t)$

$$
\begin{aligned}
\vec{A}(\vec{r}, t)=\sum_{n} & \int_{\mathrm{BZ}} \frac{d^{3} k}{(2 \pi)^{3}} \sqrt{\frac{2 \pi \xi c^{2}}{\omega_{n \vec{k}}}} * \\
& \left(\beta_{n \vec{k}}(t) \vec{A}_{n \vec{k}}(\vec{r})+\beta_{n \vec{k}}^{*}(t) \vec{A}_{n \vec{k}}^{*}(\vec{r})\right),
\end{aligned}
$$

where the time evolution of the free field is described by $\beta_{n \vec{k}}(t)=\beta_{n \vec{k}}(0) e^{-\imath \omega_{n \vec{k}} t}$. The field modes $\vec{A}_{n \vec{k}}(\vec{r})$ obey

$$
\nabla \times \nabla \times \vec{A}_{n \vec{k}}(\vec{r})=\frac{\omega_{n \vec{k}}^{2}}{c^{2}} \epsilon_{p}(\vec{r}) \vec{A}_{n \vec{k}}(\vec{r})
$$

which is the same equation as that for the electric field modes $\vec{E}_{n \vec{k}}(\vec{r})$ of Eq. (A11). We now choose the normalization of $\vec{A}_{n \vec{k}}$ such that

$$
\begin{gathered}
\int d^{3} r \epsilon_{p}(\vec{r}) \vec{A}_{n \vec{k}}(\vec{r}) \cdot \vec{A}_{m \vec{k}^{\prime}}(\vec{r})=\delta_{n m} \delta\left(\vec{k}-\vec{k}^{\prime}\right), \\
\int d^{3} r\left(\nabla \times \vec{A}_{n \vec{k}}(\vec{r})\right) \cdot\left(\nabla \times \vec{A}_{m \vec{k}^{\prime}}(\vec{r})\right)= \\
\frac{\omega_{n \vec{k}}^{2}}{c^{2}} \delta_{n m} \delta\left(\vec{k}-\vec{k}^{\prime}\right)
\end{gathered}
$$

This also fixes the normalization in Eqs. A12 and (A13). As a consequence, the total electric and magnetic field are now given by

$$
\begin{aligned}
& \vec{E}(\vec{r}, t)=\imath \sum_{n} \int_{\mathrm{BZ}} \frac{d^{3} k}{(2 \pi)^{3}} \sqrt{\frac{2 \pi \xi c^{2}}{\omega_{n \vec{k}}}} * \\
&\left(\beta_{n \vec{k}}(t) \vec{E}_{n \vec{k}}(\vec{r})-\beta_{n \vec{k}}^{*}(t) \vec{E}_{n \vec{k}}^{*}(\vec{r})\right), \\
& \vec{H}(\vec{r}, t)=\sum_{n} \int_{\mathrm{BZ}} \frac{d^{3} k}{(2 \pi)^{3}} \sqrt{\frac{2 \pi \xi c^{2}}{\omega_{n \vec{k}}}} * \\
&\left(\beta_{n \vec{k}}(t) \vec{H}_{n \vec{k}}(\vec{r})+\beta_{n \vec{k}}^{*}(t) \vec{H}_{n \vec{k}}^{*}(\vec{r})\right),
\end{aligned}
$$


where we have re-introduced the electric and magnetic field modes, $\vec{E}_{n \vec{k}}(\vec{r})=\left(\omega_{n \vec{k}} / c\right) \vec{A}_{n \vec{k}}(\vec{r})$ and $\vec{H}_{n \vec{k}}(\vec{r})=\nabla \times$ $\vec{A}_{n \vec{k}}(\vec{r})$, respectively. Eqs. A20) and (A21) finally lead us to the free field Hamiltonian

$$
H_{\mathrm{res}}=\sum_{n} \int_{\mathrm{BZ}} d^{3} k \xi \omega_{n \vec{k}}\left|\beta_{n \vec{k}}\right|^{2}
$$

The only nonzero Poisson brackets are $\left\{\beta_{n \vec{k}}, \beta_{n \vec{k}}^{*}\right\}=\imath / w$.

\section{Radiating dipole embedded in a Photonic Crystal}

We consider the insertion of a point dipole into a PBG structure at a prescribed location $\vec{r}_{0}$. The free dipole oscillator is described by the Hamiltonian $H_{\text {dip }}$

$$
H_{\text {dip }}=\frac{L \dot{q}^{2}}{2 L}+\frac{1}{2} L \omega_{0}^{2} q^{2}=\xi \omega_{0}|\alpha|^{2},
$$

where the dipole's natural frequency is $\omega_{0}=1 / L C$ and the complex oscillator amplitude $\alpha$ is given in terms of the charge $q$ and "current" $L \dot{q}$ as $\alpha(t)=q(t) \sqrt{L \omega_{0} / 2 w}+$ $\imath(L \dot{q}(t)) / \sqrt{2 \xi L \omega_{0}}$, with Poisson brackets $\left\{\alpha, \alpha^{*}\right\}=\imath / \xi$. The point dipole couples to the electric field via its dipole moment $d(t)=a q(t)$ with orientation $\hat{d}$, which yields the interaction energy

$$
H_{\mathrm{int}}=-a q(t)\left(\hat{d} \cdot \vec{E}\left(\vec{r}_{0}, t\right)\right) .
$$

In the rotating wave approximation to the interaction term, the final minimal coupling Hamiltonian for a radiating dipole in a $\mathrm{PC}$ is

$$
H=H_{\text {dip }}+H_{\text {res }}+H_{\text {ct }}+H_{\text {int }} .
$$

Collecting all the above results we obtain

$$
\begin{aligned}
H= & \xi \omega_{0}|\alpha|^{2}+\sum_{\mu} \xi \omega_{\mu}\left|\beta_{\mu}\right|^{2}+H_{\mathrm{ct}} \\
& -\imath \xi \sum_{\mu}\left(\alpha^{*} g_{\mu}^{*} \beta_{\mu}-\alpha g_{\mu} \beta_{\mu}^{*}\right) .
\end{aligned}
$$

Here, we have introduced the symbolic index $\mu \equiv(n \vec{k})$ and the coupling constants $g_{\mu}$

$$
g_{\mu} \equiv g_{\mu}\left(\vec{r}_{0}\right)=a c \sqrt{\frac{\pi}{L \omega_{0} \omega_{\mu}}}\left(\hat{d} \cdot \vec{E}_{\mu}^{*}\left(\vec{r}_{0}\right)\right) .
$$

In addition, in Eq. A25 we have introduced a mass renormalization counter term, $H_{\mathrm{ct}}=-\xi \Delta|\alpha|^{2}$ in order to cancel unphysical UV-divergent terms of our nonrelativistic theory, as discussed in the main text. For completeness, we list here only the nonzero Poisson brackets and initial values for an initially excited radiating dipole coupled to the Bloch waves of a PC. This, together with the Hamilton function $H$ in Eq. (A25) completely defines our problem:

$$
\left\{\alpha, \alpha^{*}\right\}=\left\{\beta_{\mu}, \beta_{\mu}^{*}\right\}=\frac{\imath}{\xi},
$$

where $\alpha(0)=1$ and $\beta_{\mu}(0)=0$ for all $\mu$.

\section{APPENDIX B: MODEL DISPERSION RELATION AND DENSITY OF STATES}

A particularly stringent test of our approach's ability to describe the dynamics of a radiating dipole in a PC comes from its application to a dipole coupled to a 3D isotropic photon dispersion model for the electromagnetic reservoir. In this model, the coherent scattering condition that characterizes the photonic band edge is assumed to occur at the same frequency for all directions of propagation. Clearly this is not the case in a real crystal, whose Brillouin zone cannot have full rotational symmetry. As a result, the isotropic model overestimates the electromagnetic modes available at a band-edge, so that, for example, near the upper photonic band edge at frequency $\omega_{u}$, the corresponding DOS exhibits a divergence of the form $N(\omega) \propto 1 / \sqrt{\omega-\omega_{u}}$. Conversely, for large frequencies $\left(\omega \gg \omega_{u}\right)$ the DOS will exhibit a UV-divergence, i.e., $N(\omega) \propto \omega^{2}$, as is the case in free space. More realistic LDOS' coming from full photonic band structure computations do not suffer from the pathological band edge divergence of the isotropic model. However, by solving the model of a 3D isotropic photonic band gap, we make contact with previous results based on the isotropic model in the effective mass approximation [3].

Consider a 1D photonic dispersion relation in the extended zone scheme. In order to describe a PBG at wave number $k_{0}$ with central frequency $\omega_{c}=c k_{0}=\left(\omega_{u}+\omega_{l}\right) / 2$ and upper and lower band edge at $\omega_{u}$ and $\omega_{l}$, respectively, we use the following Ansatz

$\omega(k)=\left\{\begin{array}{ll}\omega_{+}+c_{+} \sqrt{\left(k-k_{0}\right)^{2}+\gamma_{+}^{2}} & \text { for } k>0 \\ \omega_{-}+c_{-} \sqrt{\left(k-k_{0}\right)^{2}+\gamma_{-}^{2}} & \text { for } k<0\end{array}\right.$.

Using the requirements $\omega(k=0)=0, \omega\left(k=k_{0}-0_{+}\right)=$ $\omega_{l}, \omega\left(k=k_{0}+0_{+}\right)=\omega_{u}, \partial_{k} \omega(k=0)=\partial_{k} \omega(k \rightarrow \infty)=c$, and $\partial_{k} \omega\left(k=k_{0}-0_{+}\right)=\partial_{k} \omega\left(k=k_{0}+0_{+}\right)=0$, the unknown parameters in (B1) can easily be expressed in terms of a single parameter $\eta=\omega_{l} / \omega_{c}, 1 / 2<\eta \leq 1$ that describes the size of the photonic bandgap, giving: $\omega_{+}=\omega_{c}, c_{+}=c, \gamma_{+}=k_{0}(1-\eta), \omega_{-}=\omega_{c}\left(\eta^{2}\right) /(2 \eta-1)$, $c_{-}=c \eta / \sqrt{2 \eta-1}$, and $\gamma_{-}=k_{0}(1-\eta) / \sqrt{2 \eta-1}$.

From the dispersion relation (B1), the corresponding DOS, i. e., $N(\omega)=\int d^{3} k \delta(\omega-\omega(k))$ is given by

$$
N_{m}(\omega)=\left\{\begin{array}{c}
4 \pi c_{-}^{2} \frac{\left[k_{0}-\sqrt{\left(\omega-\omega_{-}\right)^{2} / c_{-}^{2}-\gamma_{-}^{2}}\right]^{2}\left(\omega_{-} \omega\right)}{\sqrt{\left(\omega-\omega_{-}\right)^{2} / c_{-}^{2}-\gamma_{-}^{2}}} \\
\text { for } 0 \leq \omega \leq \omega_{l} \\
4 \pi c_{+}^{2} \frac{\left[k_{0}+\sqrt{\left(\omega-\omega_{+}\right)^{2} / c_{+}^{2}-\gamma_{+}^{2}}\right]^{2}\left(\omega-\omega_{+}\right)}{\sqrt{\left(\omega-\omega_{+}\right)^{2} / c+-_{-} \gamma_{+}^{2}}} \\
\text { for } \omega_{u} \leq \omega<\infty
\end{array}\right.
$$

For sufficiently large gaps $(\eta \leq 0.9)$ and bare eigenfrequencies $\omega_{0}$ of the radiating dipole close to the upper band edge, it is an excellent approximation to ignore the lower branch of the photon dispersion relation, i. e., 
for $k \leq k_{0}$. The resulting DOS for this so-called threedimensional isotropic, one-sided bandgap model is shown in Fig. 1 for a value of gap width parameter $\eta=0.8$ and the gap center frequency $\omega_{c} a / 2 \pi c=0.5$. The square-root singularity at the band edge as well as the UV divergence $N_{m}(\omega) \propto \omega^{2}$ as $\omega \rightarrow \infty$ are clearly visible.

[1] C. M. Soukoulis (ed.), Photonic Band Gap Materials, Kluwer, Dordrecht (1996).

[2] S. John and J. Wang, Phys. Rev. Lett. 64, 2418 (1990), Phys. Rev. B 43, 12772 (1991).

[3] T. Quang and S. John, Phys. Rev. A 50, 1764 (1994).

[4] S. John and T. Quang, Phys. Rev. Lett. 78, 1888 (1997).

[5] S. John and T. Quang, Phys. Rev. Lett. 74, 3419 (1995).

[6] N. Vats and S. John, Phys. Rev. A 58, 4168 (1998).

[7] J.G. Flemming and S.Y. Lin, Optics Lett. 24, 49 (1999).

[8] J.E.G.J. Wijnhoven and W.L. Vos, Science 281, 802 (1998)

[9] M.S. Thijssen, R. Sprik, J.E.G.J. Wijnhoven, M. Megens, T. Narayanan, A. Lagendijk and W.L. Vos, Phys. Rev. Lett. 83, 2730 (1999).

[10] E. Özbay et al., Appl. Phys. Lett. 67, 1969 (1995).

[11] P. Ullersma, Physica 32, 27 (1966).

[12] G. M. Nikolopoulos, S. Bay, and P. Lambropoulos, Phys. Rev. A 60, 5079 (1999).

[13] L. Allen and J. H. Eberly, Optical resonance and two-level atoms, Dover Publications, 1987

[14] H. A. Bethe, Phys. Rev. 72, 339 (1947)

[15] K. Busch and S. John, Phys. Rev. E 58, 3896 (1998)

[16] R. Sprik, B. A. van Tiggelen, and A. Lagendik, Europhys. Lett. 35, 265 (1996)

[17] K.-M. Ho, C. T. Chan, and C.M. Soukoulis, Phys. Rev. Lett. 65, 3152 (1990)

[18] K. Busch and S. John, Phys. Rev. Lett. 83, 967 (1999)

[19] M. Brune, F. Schmidt-Kaler, A. Maali, J. Dreyer, E. Hagley, J. M. Raimond, and S. Haroche, Phys. Rev. Lett. 76, 1800 (1996).

[20] H. J. Carmichael, P. Kochan, B. C. Sanders, Phys. Rev. Lett. 77, 631 (1996).

[21] R. Glauber amd M. Lewenstein, Phys. Rev. A 43, 467 (1991)

[22] G. Kweon and N. M. Lawandy, Opt. Comm. 118, 388 (1995) 
Figure 1: The DOS for the three-dimensional, isotropic one-sided bandgap model of a PC. The parameters (see appendix B) are $\eta=0.8$ and $\omega_{c} a / 2 \pi c=0.5$.

Figure 2: The radiation dynamics resulting from the three-dimensional, isotropic one-sided bandgap model DOS as shown in Fig. 1 for various values of the bare dipole oscillator frequency $\omega_{0}$ relative to the upper photonic bandedge $\omega_{u}$. The photonic bandedge is siutated at $\omega_{u} a / 2 \pi c=0.6$ and the bare dipole oscillator frequencies are (a) $\omega_{0} a / 2 \pi c=0.58$, (b) $\omega_{0} a / 2 \pi c=0.595$, (c) $\omega_{0} a / 2 \pi c=0.599$, (d) $\omega_{0} a / 2 \pi c=0.6$, (e) $\omega_{0} a / 2 \pi c=$ 0.601 , (f) $\omega_{0} a / 2 \pi c=0.605$, and (g) $\omega_{0} a / 2 \pi c=0.62$. Clearly visible are normal-mode oscillations, or vacuum Rabi oscillations, and the fractional localization of radiation near the photonic bandedge. The coupling strength has been chosen such that $g\left(\omega_{0}\right)=10^{-4}$. For frequencies deep in the photonic bandgap $\left(\omega_{0} a / 2 \pi c=0.58\right)$ and deep in the photonic conduction band $\left(\omega_{0} a / 2 \pi c=0.62\right)$ we observe negligible and exponential decay, respectively. 


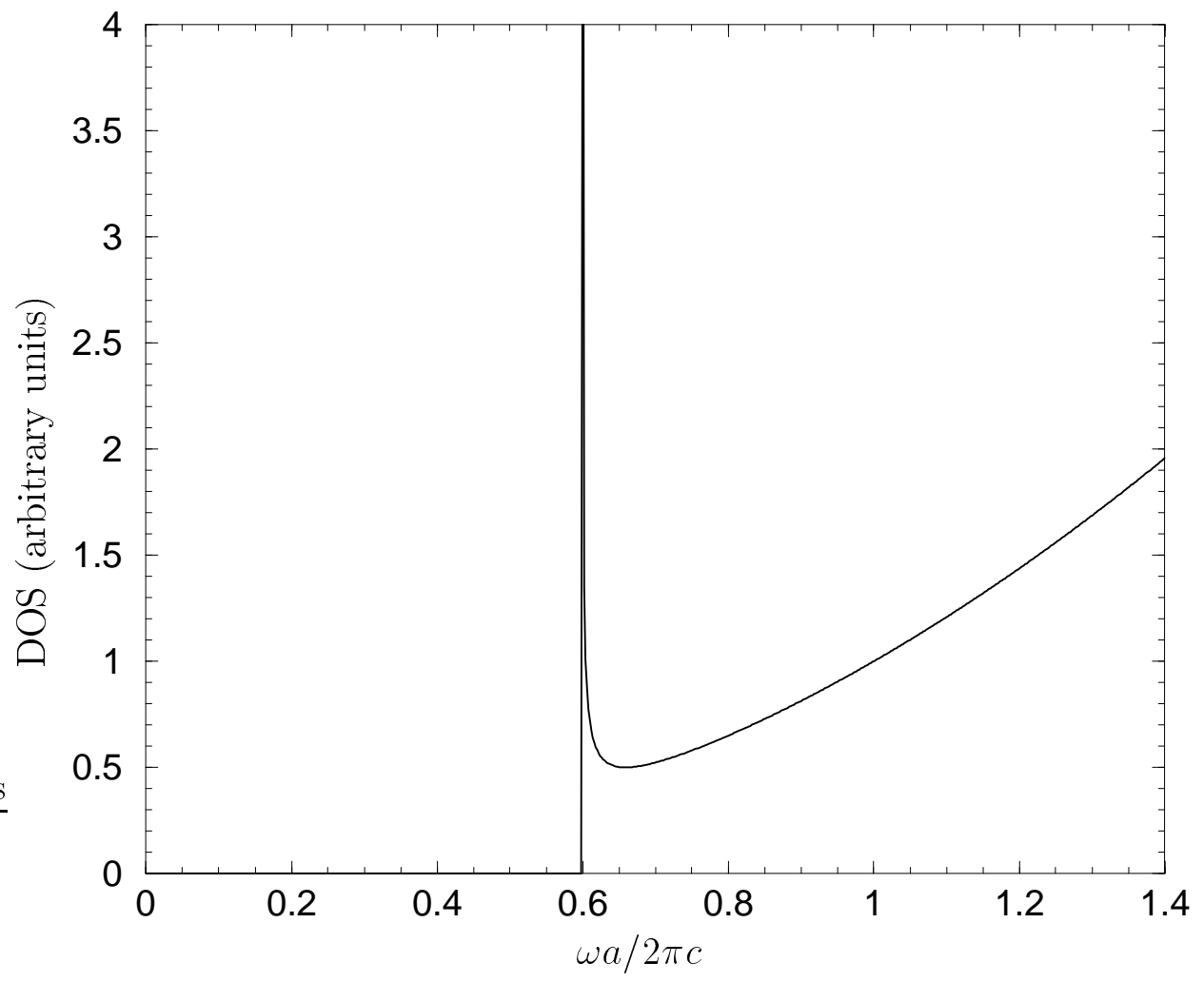

Figure 1: The DOS for the three-dimensional, isotropic one-sided bandgap model of a PC. The parameters (see appendix B) are $\eta=0.8$ and $\omega_{c} a / 2 \pi c=$ 0.5 .

Radiating Dipoles in Photonic Crystals

Kurt Busch, Nipun Vats, Sajeev John, and Barry C. Sanders 


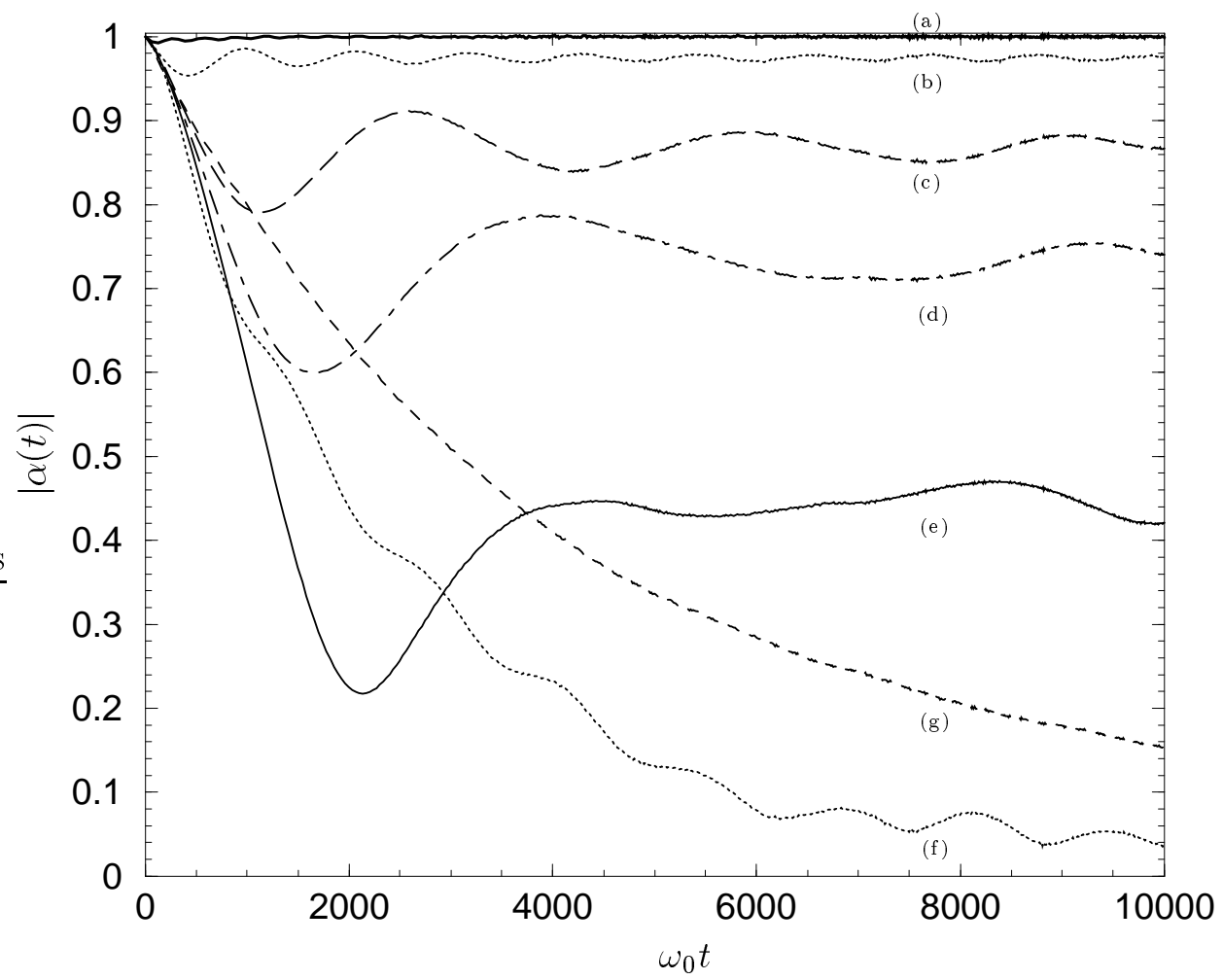

Figure 2: The radiation dynamics resulting from the three-dimensional, isotropic one-sided bandgap model DOS as shown in Fig. 1 for various values of the bare dipole oscillator frequency $\omega_{0}$ relative to the upper photonic bandedge $\omega_{u}$. The photonic bandedge is siutated at $\omega_{u} a / 2 \pi c=0.6$ and the bare dipole oscillator frequencies are (a) $\omega_{0} a / 2 \pi c=0.58$, (b) $\omega_{0} a / 2 \pi c=$ 0.595, (c) $\omega_{0} a / 2 \pi c=0.599$, (d) $\omega_{0} a / 2 \pi c=0.6$, (e) $\omega_{0} a / 2 \pi c=0.601$, (f) $\omega_{0} a / 2 \pi c=0.605$, and $(\mathrm{g}) \omega_{0} a / 2 \pi c=0.62$. Clearly visible are normalmode oscillations, or vacuum Rabi oscillations, and the fractional localization of radiation near the photonic bandedge. The coupling strength has been chosen such that $g\left(\omega_{0}\right)=10^{-4}$. For frequencies deep in the photonic bandgap $\left(\omega_{0} a / 2 \pi c=0.58\right)$ and deep in the photonic conduction band $\left(\omega_{0} a / 2 \pi c=0.62\right)$ we observe negligible and exponential decay, respectively.

Radiating Dipoles in Photonic Crystals

Kurt Busch, Nipun Vats, Sajeev John, and Barry C. Sanders 